\title{
Number Operator on Functionals of Discrete-Time Normal Noises
}

\author{
Xiling Zhang, Caishi Wang, Cuiyun Zhang, Fangqing Zhang
}

\begin{abstract}
Let $Z$ be a discrete-time normal noise that has the chaotic representation property. In this paper, we show that the number operator in the space of square integrable functionals of $Z$ can be extended to a continuous operator on the generalized functional space of $Z$.
\end{abstract}

Index Terms - Discrete-time normal noise, Generalized functional, Fock transform, Number operator.

$\operatorname{MSC}(2010):-60 \mathrm{H} 40,47 \mathrm{B38}$

\section{INTRODUCTION}

Let $Z=\left(Z_{k}\right)$ be a discrete-time normal noise, and $L^{2}(Z)$ the space of square integrable functionals of $Z$. Then the number operator $N$ in $L^{2}(Z)$ is defined as

$$
N \xi=\sum_{\sigma \in \Gamma} \# \sigma\left\langle Z_{\sigma}, \xi\right\rangle Z_{\sigma}, \quad \xi \in D o m N,
$$

where $\left\{Z_{\sigma} \mid \sigma \in \Gamma\right\}$ is the canonical orthonormal basis of $L^{2}(Z)$, \# $\sigma$ denotes the cardinality of $\sigma$ as a finite set, and $\operatorname{DomN}$ means the domain of $N$ given by

$$
\operatorname{DomN}=\left\{\left.\xi \in L^{2}(Z)\left|\sum_{\sigma \in \Gamma}(\# \sigma)^{2}\right|\left\langle Z_{\sigma}, \xi\right\rangle\right|^{2}<\infty\right\} \text {. }
$$

The operator $N$ plays an important role in functional analysis of discrete-time normal noises. For example, $-N$ generates the Ornstein-Uhlenbeck semigroup of operators on $L^{2}(Z)^{[6]}$. In a recent paper [2], $N$ is used to study the regularity of solutions to the stochastic Schrodinger equation. However, $N$ is not defined on whole $L^{2}(Z)$, namely

$\operatorname{Dom} N \neq L^{2}(Z)$, which may cause inconvenience in its application.

On the other hand, as is shown in [7], one can use the canonical orthonormal basis of $L^{2}(Z)$ to construct a nuclear space $S(Z)$ such that $S(Z)$ is densely contained in $L^{2}(Z)$. Thus, by identifying $L^{2}(Z)$ with its dual, one can get a Gel'fand triple

$$
S(Z) \subset L^{2}(Z) \subset S^{*}(Z) .
$$

where $S^{*}(Z)$ is the dual of $S(Z)$, which is endowed with

Xiling Zhang, Department of Mathematics, Northwest Normal University, Lanzhou, Gansu, China, Mobile No18368916606

Caishi Wang, Department of Mathematics, Northwest Normal University, Lanzhou, Gansu, China, Mobile No13919113627

Cuiyun Zhang, Department of Mathematics, Northwest Normal University, Lanzhou, Gansu, China, Mobile No18909406395 the strong topology, which can not be induced by any norm [3] . As usual, $S(Z)$ is called the testing functional space of $Z$, while $S^{*}(Z)$ is called the generalized functional space of $Z$. It turns out [4] that the generalized functional space $S^{*}(Z)$ can accommodate many quantities of theoretical interest that can not be covered by $L^{2}(Z)$.

In this paper, we would like to extend the number operator $N$ to generalized functionals of $Z$. More precisely, we will define the number operator on the generalized functional space $S^{*}(Z)$.

Throughout this paper, $N$ designates the set of all nonnegative integers and $\Gamma$ the finite power set of $N$, namely

$$
\Gamma=\{\sigma \mid \sigma \subset N, \#(\sigma)<\infty\},
$$

where $\# \sigma$ means the cardinality of $\sigma$ as a finite set. If $k \in N$ and $\sigma \in \Gamma$, then we simply write $\sigma \bigcup k$ for $\sigma \bigcup\{k\}$. Similarly, we use $\sigma \backslash k$.

\section{PRELIMINARY}

In what follows, we always assume that $(\Omega, F, P)$ is a given probability space. We use $E$ to mean the expectation with respect to $P$. As usual, $L^{2}(\Omega, F, P)$ denotes the Hilbert space of square integrable complex-valued measurable functions on $(\Omega, F, P)$. We use $\langle\cdot, \cdot\rangle$ and $\|\cdot\|$ to mean the inner product and norm of $L^{2}(\Omega, F, P)$, respectively. By convention, $\langle\cdot \cdot\rangle$ is conjugate-linear in its first argument and linear in its second argument.

Define2.1A sequence $Z=\left(Z_{n}\right)_{n \in N}$ of integrable random variables on $(\Omega, F, P)$ is called a discrete-time normal noise if it satisfies:

(i) $E\left[Z_{n} \mid F_{n-1}\right]=0$ for $n \geq 0$;

(ii) $E\left[Z_{n}^{2} \mid F_{n-1}\right]=1$ for $n \geq 0$.

Here $\left.F_{-1}=\{\phi, \Omega\}_{1}\right)_{n}=\sigma\left(Z_{k} ; 0 \leq k \leq n\right)$ for $n \in N$ and $E\left[\cdot \mid F_{n}\right]$ means the conditional expectation given $F_{n}$.

For a discrete-time normal noise $Z=\left(Z_{n}\right)_{n \in N}$ on $(\Omega, F, P)$, one can construct a corresponding family $\left\{Z_{\sigma} \mid \sigma \in \Gamma\right\}$ of random variables on $(\Omega, F, P)$ in the following manner 


$$
Z_{\phi}=1 \quad \text { and } \quad Z_{\sigma}=\prod_{i \in \sigma} Z_{\mathrm{i}}, \quad \sigma \in \Gamma, \quad \sigma \neq \phi
$$

We call $\left\{Z_{\sigma} \mid \sigma \in \Gamma\right\}$ the canonical functional system of Z.

Lemma $2.1^{[6]}$ Let $Z=\left(Z_{n}\right)_{n \in N}$ be a discrete-time normal noise on $(\Omega, F, P)$.Then its canonical functional system $\left\{Z_{\sigma} \mid \sigma \in \Gamma\right\}$ forms a countable orthonormal system in $L^{2}(\Omega, F, P)$.

Let $F_{\infty}=\sigma\left(Z_{n} ; n \in N\right)$ be the $\sigma$-field over $\Omega$ generated by a discrete-time normal noise $Z=\left(Z_{n}\right)_{n \in N}$ on $(\Omega, F, P)$. Then the canonical functional system $\left\{Z_{\sigma} \mid \sigma \in \Gamma\right\}$ is also a countable orthonormal system in the space $L^{2}\left(\Omega, F_{\infty}, P\right)$ of square integrable complex-valued measurable functions on $\left(\Omega, F_{\infty}, P\right)$.

In the literature, $F_{\infty}$-measurable functions on $\Omega$ are also known as functionals of $Z$. Thus elements of $L^{2}\left(\Omega, F_{\infty}, P\right)$ are naturally called square integrable

Define 2.2 A discrete-time normal noise $Z=\left(Z_{n}\right)_{n \in N}$ on $(\Omega, F, P)$ is said to have the chaotic representation property if its canonical functional system $\left\{Z_{\sigma} \mid \sigma \in \Gamma\right\}$ is total in $L^{2}\left(\Omega, F_{\infty}, P\right)$, where $F_{\infty}=\sigma\left(Z_{n} ; n \in N\right)$.

Thus, if a discrete-time normal noise $Z$ has the chaotic representation property, then its canonical functional system $\left\{Z_{\sigma} \mid \sigma \in \Gamma\right\}$ is actually an orthonormal basis of $L^{2}\left(\Omega, F_{\infty}, P\right)$.

From now on, we always assume that $Z=\left(Z_{n}\right)_{n \in N}$ is a given discrete-time normal noise on $(\Omega, F, P)$ that has the chaotic representation property.

For brevity, we use $L^{2}(Z)$ to denote the space of square integrable functionals of $Z$, namely

$$
L^{2}(Z)=L^{2}\left(\Omega, F_{\infty}, P\right),
$$

where $F_{\infty}=\sigma\left(Z_{n} ; n \in N\right)$. For $k \geq 0$, we denote by $F_{k}$ the $\sigma$ - field generated by $\left(Z_{j} ; 0 \leq j \leq k\right)$, namely

$$
F_{k}=\sigma\left(Z_{j} ; 0 \leq j \leq k\right) \text {. }
$$

We note that $L^{2}(Z)$ shares the same inner product $\langle\cdot, \cdot\rangle$ and norm $\|$.$\| with L^{2}(\Omega, F, P)$, and moreover the canonical functional system $\left\{Z_{\sigma} \mid \sigma \in \Gamma\right\}$ of $Z$ forms a countable orthonormal basis for $L^{2}(Z)$, which we call the canonical orthonormal basis of $L^{2}(Z)$.

Lemma $2.2^{[7]}$ Let $\sigma \mapsto \lambda_{\sigma}$ be the $N$-valued function on $\Gamma$ given by $\lambda_{\sigma}=\left\{\begin{array}{l}\prod_{k \in \sigma}(k+()), \zeta \neq \phi, \sigma \in \Gamma ; \\ 1, \sigma=\phi, \sigma \in \Gamma .\end{array}\right.$

Then, for $p>1$, the positive term series $\sum_{\sigma \in \Gamma} \lambda_{\sigma}^{-p}$

converges and moreover

$$
\sum_{\sigma \in \Gamma} \lambda_{\sigma}^{-p} \leq \exp \left[\sum_{k=1}^{\infty} k^{-p}\right]<\infty
$$

Using the $N$-valued function defined by (2.1), we can construct a chain of Hilbert spaces consisting of functionals of $Z$ as follows. For $p \geq 0$, we put

$$
S_{p}(Z)=\left\{\left.\xi \in L^{2}(Z)\left|\sum_{\sigma \in \Gamma} \lambda_{\sigma}^{2 p}\right|\left\langle Z_{\sigma}, \xi\right\rangle\right|^{2}<\infty\right\}
$$

and define

$$
\langle\xi, \eta\rangle_{p}=\sum_{\sigma \in \Gamma} \lambda_{\sigma}^{2 p} \overline{\left\langle Z_{\sigma}, \xi\right\rangle}\left\langle Z_{\sigma}, \eta\right\rangle, \quad \xi, \eta \in S_{p}(Z) .
$$

It is not hard to check that, with $\langle\cdot,\rangle_{p}$ as the inner product, $S_{p}(Z)$ becomes a Hilbert space. We write $\|\xi\|_{p}=\sqrt{\langle\xi, \xi\rangle}$ for $\xi \in S_{p}(Z)$. Clearly, it holds that

$$
\|\xi\|_{p}^{2}=\sum_{\sigma \in \Gamma} \lambda_{\sigma}^{2 p}\left|\left\langle Z_{\sigma}, \xi\right\rangle\right|^{2}, \quad \xi \in S_{p}(Z)
$$

Lemma2.3 $\left.{ }^{[4,7]}\right]$ For $p \geq 0,\left\{Z_{\sigma} \mid \sigma \in \Gamma\right\} \subset S_{p}(Z)$ and moreover the system $\left\{\lambda_{\sigma}^{-p} Z_{\sigma} \mid \sigma \in \Gamma\right\}$ forms an orthonormal basis for $S_{p}(Z)$.

It is easy to see that $\lambda_{\sigma} \geq 1$ for all $\sigma \in \Gamma$. This implies that $\quad\|\cdot\|_{p} \leq\|\cdot\|_{q} \quad$ and $\quad S_{q}(Z) \subset S_{p}(Z) \quad$ whenever $0 \leq p \leq q$. Thus we actually get a chain of Hilbert spaces of functionals of $Z$ :

$\cdots \subset S_{p+1}(Z) \subset S_{p}(Z) \subset \cdots \subset S_{1}(Z) \subset S_{0}(Z)=L^{2}(Z)$

We now put

$$
S(Z)=\bigcap_{p=0}^{\infty} S_{p}(Z)
$$

and endow it with the topology generated by the norm sequence $\left\{\|\cdot\|_{p}\right\}_{p \geq 0}$. Note that, for each $p \geq 0, S_{p}(Z)$ is just the completion of $S(Z)$ with respect to $\|\cdot\|_{p}$ Thus $S(Z)$ is a countably-Hilbert space ${ }^{[1,3]}$. The next lemma, however, shows that $S(Z)$ even has a much better property.

Lemma2.4 ${ }^{[4,7]}$ The space $S(Z)$ is a nuclear space, namely for any $p \geq 0$, there exists $q>p$ such that the inclusion mapping $i_{p q}: S_{q}(Z) \rightarrow S_{p}(Z)$ defined by $i_{p q}(\xi)=\xi$ is a Hilbert-Schmidt operator.

For $p \geq 0$, we denote by $S_{p}^{*}(Z)$ the dual of $S_{p}(Z)$ and $\|\cdot\|_{-p}$ the norm of $S_{p}^{*}(Z)$. Then $S_{p}^{*}(Z) \subset S_{q}^{*}(Z)$ and 
$\|\cdot\|_{-p} \geq\|\cdot\|_{-q}$ whenever $0 \leq p \leq q$. The lemma below is then an immediate consequence of the general theory of countably-Hilbert spaces (see, e.g., [1] or [3]).

Lemma $2.5^{[4,7]}$ Let $S^{*}(Z)$ be the dual of $S(Z)$ and endow it with the strong topology. Then

$$
S^{*}(Z)=\bigcup_{p=0}^{\infty} S_{p}^{*}(Z)
$$

and moreover the inductive limit topology over $S^{*}(Z)$ given by space sequence $\left\{S_{p}^{*}(Z)\right\}_{p \geq 0}$ coincides with the strong topology.

We mention that, by identifying $L^{2}(Z)$ with its dual, one comes to a Gel'fand triple

$$
S(Z) \subset L^{2}(Z) \subset S^{*}(Z)
$$

which we refer to as the Gel'fand triple associated with the discrete-time normal noise $Z$.

Lemma $2.6^{[4]}$ The system $\left\{Z_{\sigma} \mid \sigma \in \Gamma\right\}$ is contained in $S(Z)$ and moreover it forms a basis for $S(Z)$ in the sense that

$$
\xi=\sum_{\sigma \in \Gamma}\left\langle Z_{\sigma}, \xi\right\rangle Z_{\sigma}, \quad \xi \in S(Z)
$$

where $\langle\cdot$,$\rangle is the inner product of L^{2}(Z)$ and the series converges in the topology of $S(Z)$.

Define 2.3 ${ }^{[4,7]}$ Elements of $S^{*}(Z)$ are called generalized functionals of $Z$, while elements of $S(Z)$ are called testing functionals of $Z$.

Thus, $S^{*}(Z)$ and $S(Z)$ can be accordingly called the generalized functional space and the testing functional space of $Z$, respectively. It turns out [4] that $S^{*}(Z)$ can accommodate many quantities of theoretical interest that can not be covered by $L^{2}(Z)$.

In the following, we denote by $\langle\langle\cdot, \cdot\rangle\rangle$ the canonical bilinear form on $S^{*}(Z) \times S(Z)$ given by

$$
\langle\langle\Phi, \xi\rangle\rangle=\Phi(\xi), \quad \Phi \in S^{*}(Z), \xi \in S(Z) .
$$

Note that $\langle\langle\cdot, \cdot\rangle\rangle$ is different from the inner product $\langle\cdot, \cdot\rangle$ of $L^{2}(Z)$.

Define 2.4 ${ }^{[4]}$ For $\Phi \in S^{*}(Z)$, its Fock transform is the function $\bar{\Phi}$ on $\Gamma$ given by

$$
\bar{\Phi}(\sigma)=\left\langle\left\langle\Phi, Z_{\sigma}\right\rangle\right\rangle, \quad \sigma \in \Gamma
$$

where $\langle\langle\cdot, \cdot\rangle\rangle$ is the canonical bilinear form.

It is easy to verify that, for $\Phi, \Psi \in S^{*}(Z), \Phi=\Psi$ if and only if $\bar{\Phi}=\bar{\Psi}$. Thus a generalized functional of $Z$ is completely determined by its Fock transform. The following theorem characterizes generalized functionals of $Z$ through their Fock transforms.

Lemma $2.7^{[4]}$ Let $F$ be a function on $\Gamma$. Then $F$ is the Fock transform of an element $\Phi$ of $S^{*}(Z)$ if and only if it

satisfies

$$
|F(\sigma)| \leq C \lambda_{\sigma}^{p}, \quad \sigma \in \Gamma
$$

for $q>p+\frac{1}{2}$, one has

$$
\|\Phi\|_{-q} \leq C\left[\sum_{\sigma \in \Gamma} \lambda_{\sigma}^{-2(q-p)}\right]^{\frac{1}{2}}
$$

and in particular $\Phi \in S_{q}^{*}(Z)$

Remark2.1There.10)xists a continuous linear mapping $R: L^{2}(Z) \rightarrow S^{*}(Z)$ such that

$$
\langle\langle R \eta, \xi\rangle\rangle=\langle\eta, \xi\rangle, \quad \eta \in L^{2}(Z), \xi \in S(Z) .
$$

where $\langle\langle\cdot, \cdot\rangle\rangle$ is the canonical bilinear form on $S^{*}(Z) \times S(Z)_{(2 \mathrm{~W} \text { (Y) }}$ call $R$ the Riesz mapping

\section{MAIN RESULT}

In this section, we define our number operator on the generalized functional space $S^{*}(Z)$ and show its links with the number operator $N$ in $L^{2}(Z)$. Our main tool is the Fock transforms of generalized functionals of $Z$.

Recall that $\# \sigma$ means the cardinality of $\sigma$ as a finite set. The following lemma gives an inequality concerning $\# \sigma$, which will be used later.

Lemma 3.1For all $\sigma \in \Gamma$, it holds that $\# \sigma \leq \lambda_{\sigma}$.

Proof. Let $\sigma \in \Gamma$. Clearly, $\# \sigma \leq \lambda_{\sigma}$ holds for the case of $\sigma=\phi$. For the case of $\sigma \neq \phi$, by assuming $\sigma=\left\{k_{1}, k_{2}, \cdots, k_{n}\right\}$ with $k_{1}<k_{2}<\cdots k_{n}$.we have

$\left.\lambda_{\sigma}=\left(k_{1}+1\right)\left(k_{2}^{2}+12\right)\right) \cdots\left(k_{n}+1\right) \geq 1 \times 2 \times \cdots \times n \geq n=\# \sigma$

This completes the proof.

Proposition3.2There exists a linear operator $n: S^{*}(Z) \rightarrow S^{*}(Z)$ such that

$\overline{n \Phi}(\sigma)=\# \sigma \bar{\Phi}(\sigma), \quad \Phi \in S^{*}(Z), \sigma \in \Gamma$

where $\bar{\Phi}$ and $\overline{n \Phi}$ are Fock transforms of $\Phi$ and $n \Phi$, respectively.

Proof. Let $\Phi \in S^{*}(Z)$. Then, by Lemma 2.7, there exist constants $C \geq 0$ and $p \geq 0$ such that 


$$
|\bar{\Phi}(\sigma)| \leq C \lambda_{\sigma}^{p}, \quad \sigma \in \Gamma
$$

which together with Lemma 3.1 gives

$$
|\# \sigma \bar{\Phi}(\sigma)| \leq C \lambda_{\sigma}^{p+1}, \quad \sigma \in \Gamma
$$

which together Lemma 2.7 implies that there exists a unique generalized functional $\Phi^{\prime} \in S^{*}(Z)$ such that

$$
\overline{\Phi^{\prime}}(\sigma)=\# \sigma \bar{\Phi}(\sigma), \quad \sigma \in \Gamma \text {. }
$$

Thus we have a mapping $\Phi \rightarrow \Phi^{\prime}$ from $S^{*}(Z)$ into itself, which we denote by $n$, namely

$$
n \Phi=\Phi^{\prime}, \Phi \in S^{*}(Z)
$$

Obviously $n$ satisfies (3.1). Now let $\Phi_{1}, \Phi_{2} \in S^{*}(Z)$ and $\alpha_{1}, \alpha_{2}$ be any complex number. Then, by letting $\Psi=\alpha_{1} \Phi_{1}+\alpha_{2} \Phi_{2}$, we have

$$
\begin{aligned}
\overline{n \Psi}(\sigma) & =\# \sigma \hat{\Psi}(\sigma) \\
& =\alpha_{1} \# \sigma \overline{\Phi_{1}}(\sigma)+\alpha_{2} \# \sigma \overline{\Phi_{2}}(\sigma) \\
& =\alpha_{1} \overline{n \Phi_{1}}(\sigma)+\alpha_{2} \overline{n \Phi_{2}}(\sigma), \quad \sigma \in \Gamma
\end{aligned}
$$

which implies that $n \Psi=\alpha_{1} n \Phi_{1}+\alpha_{2} n \Phi_{2}$. Thus $n$ is a linear operator on $S^{*}(Z)$.

Proposition 3.3The operator $n$ is continuous on $S^{*}(Z)$. Proof. We need only to show that the composition $n \circ j_{p}: S_{p}^{*}(Z) \rightarrow S^{*}(Z)$ is continuous for each $p \geq 0$, where $j_{p}: S_{p}^{*}(Z) \rightarrow S^{*}(Z)$ denotes the natural embedding.

Let $p \geq 0$ and take $q>p+\frac{3}{2}$. Then, for each $\Phi \in S_{p}^{*}(Z)$, it follows from the inequality $\# \sigma \leq \lambda_{\sigma}, \sigma \in \Gamma$ that

$$
\begin{aligned}
& |\overline{n \Phi}(\sigma)|=\# \sigma|\bar{\Phi}(\sigma)|=\# \sigma\left|\left\langle\left\langle\Phi, Z_{\sigma}\right\rangle\right\rangle\right| \leq \lambda_{\sigma}\|\Phi\|_{-P}\left\|Z_{\sigma}\right\|_{p} \\
& =\|\Phi\|_{-p} \lambda_{\sigma}^{p+1}, \forall \sigma \in \Gamma .
\end{aligned}
$$

which together with the characterization theorem (Lemma2.7) implies that $n \Phi \in S_{q}^{*}(Z)$ and

$$
\|n \Phi\|_{-q} \leq\left[\sum_{\sigma \in \Gamma} \lambda_{\sigma}^{-2(q-p-1)}\right]^{\frac{1}{2}}\|\Phi\|_{-p} .
$$

Thus, $\forall \Phi \in S_{p}^{*}(Z)$, we have $n \circ j_{p}(\Phi)=n \Phi \in S_{q}^{*}(Z)$, and moreover

$$
\left\|n \circ j_{p}(\Phi)\right\|_{-q} \leq\left[\sum_{\sigma \in \Gamma} \lambda_{\sigma}^{-2(q-p-1)}\right]^{\frac{1}{2}}\|\Phi\|_{-p} .
$$

which means that $n \circ j_{p}$ is a continuous linear operator from $S_{p}^{*}(Z)$ to $S_{p}^{*}(Z)$, hence a continuous linear operator from $S_{p}^{*}(Z)$ to $S^{*}(Z)$.

From Proposition 3.2 and Proposition 3.3, we see that $n$ is actually a continuous linear operator from $S^{*}(Z)$ to itself.

Recall that the number operator $N$ in $L^{2}(Z)$ is defined by

$$
\begin{gathered}
N \xi=\sum_{\sigma \in \Gamma} \# \sigma\left\langle Z_{\sigma}, \xi\right\rangle Z_{\sigma}, \quad \xi \in D o m N \\
\left\{Z_{\sigma} \mid \sigma \in \Gamma\right\} \\
\text { is the canonical orthonormal basis of }
\end{gathered}
$$

yberz)

$$
\operatorname{DomN}=\left\{\begin{array}{c}
\text { and means the domain of given by } \\
\left.\left.\xi \in L^{2}(Z)\left|\sum_{\sigma \in \Gamma}(\# \sigma)^{2}\right|\left\langle Z_{\sigma}, \xi\right\rangle\right|^{2}<\infty\right\}
\end{array}\right.
$$

The next proposition then shows the link between and Proposition3.4Let $R: L^{2}(Z) \rightarrow S^{*}(Z)$ be the Riesz mapping. Then it holds that

$$
n R \xi=R N \xi, \quad \forall \xi \in D o m N .
$$

where $N$ is the number operator in $L^{2}(Z)$.

Proof. Let $\xi \in D o m N$. Then, $\forall \sigma \in \Gamma$, we have

$\overline{n R \xi}(\sigma)=\overline{n(R \xi)}(\sigma)=\# \sigma \overline{R \xi}(\sigma)=\# \sigma\left\langle\left\langle R \xi, Z_{\sigma}\right\rangle\right\rangle$

$=\# \sigma\left\langle\xi, Z_{\sigma}\right\rangle$

$\stackrel{\text { which together with }}{R N}(\sigma)=R(N \xi)(\sigma)=\left\langle\left\langle R(N \xi), Z_{\sigma}\right\rangle\right\rangle=\left\langle N \xi, Z_{\sigma}\right\rangle$

$=\left\langle\xi, N Z_{\sigma}\right\rangle=\# \sigma\left\langle\xi, Z_{\sigma}\right\rangle$ gives

$\overline{n R \xi}(\sigma)=\overline{R N \xi}(\sigma)$. Thus, $n R \xi=R N \xi$

follows from the arbitrariness of $\sigma \in \Gamma$.

Remark 3.1In view of Proposition 3.3, we may think of $n$ as the extension of the number operator $N$ to the generalized functional space $S^{*}(Z)$ and call it the number operator on

\section{ACKNOWLEDGMENT}

The author is very grateful to the anonymous referees for their valuable suggestions. This work is supported by National Natural Science Foundation of China (Grant No. 11461061 and Grant No. 11861057). 


\section{REFERENCES}

[1]J. Becnel, Equivalence of topologies and Borel fields for countably-Hilbert spaces[J].Proc.Amer.Math. Soc. 2006,134: 581-590.

[2]J. Chen and C. Wang, Linear stochastic Schrodinger equations in terms of quantum Bernoulli noises[J]. Math. Phys. 2017,58, 053510.

[3]I.M. Gelfand and N.Ya. Vilenkin, Generalized Functions vol. 4, Applications of Harmonic Analysis, Academic Press, New York, 1964

[4]C. Wang and J. Chen, Characterization theorems for generalized functionals of discrete-time normal martingale, [J]. Funct. Spaces. 2015, Art. ID 714745, 6 pages.

[5]C. Wang, S. Lin and A. Huang, Clark-Ocone formula for generalized functionals of discrete-time normal noises[J]. Funct. Spaces .2018, Art. ID 2954695.

[6]C. Wang, Y. Lu, and H. Chai, An alternative approach to Privault's discrete-time chaotic calculus[J]. Math. Anal. Appl. 2011,373 643-654.

[7]C. Wang and J. Zhang, Wick analysis for Bernoulli noise functionals [J]. Funct. Spaces. 2014, Art. ID 727341, 7 pages.

Xiling Zhang, Collage of Mathematics and Statistics, Northwest Normal University, Lanzhou, China, Mobile 86- 18368916606. 